\title{
Setting Eligibility Criteria for a Care-Coordination Benefit
}

\author{
Christine T. Cigolle, MD, MPH, ${ }^{*}$ Kenneth M. Langa, MD, PhD ${ }^{\dagger+\mathcal{S}}$ Mohammed U. Kabeto, MA, ${ }^{\dagger}$ and \\ Caroline S. Blaum, MD, MS ${ }^{\dagger / l}$
}

OBJECTIVES: To examine different clinically relevant eligibility criteria sets to determine how they differ in numbers and characteristics of individuals served.

DESIGN: Cross-sectional analysis of the 2000 wave of the Health and Retirement Study (HRS), a nationally representative longitudinal health interview survey of adults aged 50 and older.

SETTING: Population-based cohort of community-dwelling older adults, subset of an ongoing longitudinal health interview survey.

PARTICIPANTS: Adults aged 65 and older who were respondents in the 2000 wave of the HRS $(n=10,640$, representing approximately 33.6 million Medicare beneficiaries).

MEASUREMENTS: Three clinical criteria sets were examined that included different combinations of medical conditions, cognitive impairment, and activity of daily living/ instrumental activity of daily living (ADL/IADL) dependency. RESULTS: A small portion of Medicare beneficiaries (1.3$5.8 \%$ ) would be eligible for care coordination, depending on the criteria set chosen. A criteria set recently proposed by Congress (at least four severe complex medical conditions and one ADL or IADL dependency) would apply to 427,000 adults aged 65 and older in the United States. Cri-

From the Departments of *Family Medicine and "Internal Medicine and ${ }^{\ddagger}$ Institute for Social Research, University of Michigan, Ann Arbor, Michigan; $\S^{\S}$ Ann Arbor Department of Veterans Affairs Center for Practice Management and Outcomes Research, Ann Arbor, Michigan; and "Ann Arbor VA Healthcare System Geriatric Research, Education and Clinical Center, Ann Arbor, Michigan.

This work was supported by a John A. Hartford Foundation grant to the Society of General Internal Medicine (2002-0013). Dr. Cigolle was supported by the University of Michigan Institute of Gerontology Research Training Program and by the Veterans Administration Special Fellows Program in Advanced Geriatrics. Dr. Langa was supported by a Career Development Award from the National Institute on Aging (K08 AG19180) and a Paul Beeson Physician Faculty Scholars in Aging Research Award. Dr. Blaum was supported by a National Institute on Aging grant (R01 AG021493-01). The National Institute on Aging provided funding for the Health and Retirement Study (U01 AG09740), data from which were used in this study. An early version of this paper was presented at the 2003 Gerontological Society of America National Meeting, San Diego, California.

Address correspondence to Christine T. Cigolle, MD, MPH, University of Michigan Medical School, CCGCB Room 1111, 1500 East Medical Center Drive, Ann Arbor, MI 48109. E-mail: ccigolle@umich.edu

DOI: $10.1111 / j .1532-5415.2005 .00496 . x$ teria emphasizing cognitive impairment would serve an older population.

CONCLUSION: Several criteria sets for a Medicare carecoordination benefit are clinically reasonable, but different definitions of eligibility would serve different numbers and population groups of older adults. J Am Geriatr Soc 53:2051-2059, 2005.

Key words: chronic disease; cognitive impairment; activities of daily living; instrumental activities of daily living; care coordination

$\mathrm{O}$ der adults with multiple chronic diseases account for a large share of healthcare utilization and costs in the United States. ${ }^{1}$ Despite having access to complex, high-cost care, older adults with multiple chronic diseases are not well served by the present healthcare delivery system, with its fragmentation and its historical emphasis on acute care. Cost and quality shortcomings have prompted the medical and policy communities to examine alternate approaches to the care of these older adults.

One alternative is disease management, which has been characterized as "an intervention designed to manage or prevent a chronic condition using a systematic approach to care and potentially employing multiple treatment modalities." ${ }^{2}$ Yet there remains only limited evidence that disease management improves health or reduces utilization and costs in the long term and across the spectrum of diseases. ${ }^{2,3}$ Also, disease management, with its focus on a single disease, may not be the model best suited to provide care to older adults with multiple chronic diseases, cognitive impairment, and disability. For instance, studies of older adults with congestive heart failure and of those with diabetes mellitus have revealed the critical role of comorbidities in contributing to preventable hospitalizations. ${ }^{4,5}$

It has been suggested instead that some form of care management or care coordination would be a more-appropriate approach for these older adults. Research on medical care coordination is limited. For example, research on coordination of long-term care services (home and community-based) in the 1990s generally focused on coordination of personal care needs by nonmedical providers, in contrast to the present focus on coordination of multiple medical 
problems by medical providers. ${ }^{6-9}$ There are few data demonstrating that care coordination for those with multiple chronic diseases results in improved quality of care or decreased utilization and costs. Also, the ideal target population for care coordination has not been defined.

The mixed results of research on disease management and care coordination suggest that these care delivery models require further refinement before they assume a prominent place in Medicare reform. A care-coordination benefit was a key feature of two pieces of legislation introduced in Congress in 2003 but never passed. ${ }^{10-13}$ In addition, a care coordination demonstration project was included in the Senate legislation that was the basis for the Medicare Prescription Drug, Improvement, and Modernization Act of 2003 (Medicare Modernization Act), but this demonstration project was removed from the final version of the law. ${ }^{14}$ In each legislative version, the care-coordination benefit would reimburse physicians for medical care coordination services provided to those Medicare beneficiaries who met eligibility criteria. Although specific eligibility criteria for the benefit varied in the different pieces of legislation, the criteria generally consisted of some combination of complex medical conditions, cognitive impairment, and disability. Language from the original Senate version of the Medicare Modernization Act required eligible beneficiaries, first, to have at least four complex medical conditions (one of which could be cognitive impairment) and, second, to have an inability to self-manage care or to have one or more activity of daily living (ADL) or instrumental activity of daily living (IADL) impairments. The Senate bill did not specifically define "complex medical condition" but instead referred to chronic conditions that are incurable, require ongoing medical care, and may affect performance of ADLs or IADLs.

The differing legislative criteria for care coordination suggest that there has been insufficient study of clinically relevant eligibility criteria and of the numbers of Medicare beneficiaries eligible for a care-coordination benefit under different criteria. Administrative claims data, a key source of prevalence information about chronic diseases, does not provide information about cognitive impairment and ADL/ IADL dependencies; this restricts the relevant clinical information that can be considered and modeled in any proposed eligibility criteria set.

In this research, secondary data analysis of a national health interview survey that provides data not only on chronic diseases but also on cognitive impairment and ADL and IADL dependencies was performed; furthermore, this survey's determination of cognitive impairment is performance based. The research goal was to investigate numbers and characteristics of Medicare beneficiaries potentially eligible for care coordination based on alternate sets of clinically relevant eligibility criteria.

\section{METHODS}

\section{Data}

The data used in this study are from the 2000 wave of the Health and Retirement Study (HRS). The HRS is a biennial longitudinal health interview survey of a cohort of adults aged 50 and older in the United States designed to study the effect of health transitions in this group. It is based on a nationally representative multistage probability sample of households. The National Institute on Aging sponsors the HRS, and the Institute for Social Research at the University of Michigan performs it. Among longitudinal health interview surveys, it is distinctive in that it provides detailed selfreport information on chronic diseases, cognitive impairment, and task-specific disabilities, in addition to healthcare utilization and expenditures. ${ }^{15,16}$

Of the 19,580 HRS respondents interviewed in 2000, 10,640 respondents aged 65 and older and residing in the community at the time of interview were identified; they represented 33.6 million community-dwelling adults aged 65 and older in the United States that year. The HRS conducted interviews with sampled respondents and their spouses. When the eligible respondent was unable to be interviewed, often because of medical or cognitive problems, a proxy respondent $(\mathrm{n}=1,069)$ most often the spouse $(\mathrm{n}=651)$, was interviewed instead.

The Behavioral Sciences Committee institutional review board at the University of Michigan approved the HRS. The data used for this analysis contained no unique identifiers, thus assuring respondent anonymity.

\section{Variables and Their Measurement Complex Medical Conditions}

The original Senate version of the Medicare Modernization Act specified that one eligibility criterion for the care-coordination benefit was having at least four complex medical conditions. The legislation did not define or specify what constituted a complex medical condition, instead referring to chronic conditions that are incurable, require ongoing medical care, and may affect performance of ADLs or IADLs; one of the complex medical conditions could be cognitive impairment. On the basis of this definition, the following diseases surveyed in the HRS were considered to be complex medical conditions: heart disease, chronic lung disease, diabetes mellitus, cancer, musculoskeletal conditions, stroke, psychiatric problems, and cognitive impairment. Analyses were performed that included and excluded hypertension. Hypertension often requires physician visits and multiple medications, and it may contribute to disability in the presence of heart disease, stroke, and diabetes mellitus; thus, it can be considered a complex medical condition. Yet it is also reasonable not to consider hypertension a complex medical condition, for alone it is not typically associated with disability. ${ }^{17}$ Incontinence and falls were not considered to be complex medical conditions, because both are geriatric conditions that may result from multiple rather than single disease processes. ${ }^{18,19}$ The 2000 wave of the HRS does not include specific questions on diseases or conditions such as chronic kidney disease, Parkinson's, and neuropathy.

Respondents reported whether a physician had diagnosed them with each medical condition. Each condition had its own set of follow-up questions, and certain of the follow-up questions were selected to serve as indicators of the activity or severity of the condition. Because the second eligibility criterion for the care-coordination benefit was ADL/IADL dependency, activity/severity constraints that were inherently functional in nature were avoided. The medical conditions and their respective activity/severity constraints are: 
Heart disease: requiring medication for ongoing angina pectoris, for a myocardial infarction in the previous 2 years, or for congestive heart failure

Chronic lung disease: requiring medication or other treatment

Diabetes mellitus: requiring oral medication or insulin

Cancer excluding skin cancer, requiring treatment within the previous 1 to 2 years

Musculoskeletal conditions: arthritis (unspecified type) requiring medication or other treatment, joint replacement in the previous 2 years, or hip fracture in the previous 2 years

Stroke: resulting in remaining problems or requiring medication

Psychiatric problems: emotional, nervous, or psychiatric problems requiring medication or therapy

Hypertension: requiring medication

\section{Cognitive Impairment}

Cognitive impairment was evaluated in two ways: first, as one of the eight medical conditions (as in the legislation's language), and, second, as an independent eligibility criterion for a care-coordination benefit.

The HRS assesses cognitive impairment in one of two ways. For self-respondents, the presence of cognitive impairment is defined using a performance-based measure, a modified version of the Telephone Interview for Cognitive Status, a validated cognitive screening instrument patterned on the Mini-Mental State Examination ${ }^{20}$ and specifically designed for population-based studies. The questions that measure cognitive impairment in the HRS include tests of memory and reasoning and other items from a mental status questionnaire. Cognitive impairment was defined as a score of 8 or less on the 35-point cognitive scale. Researchers have previously used this cutpoint, because the proportion of people that it identifies as having serious cognitive impairment is consistent with other estimates of the prevalence of dementia. ${ }^{21-23}$ Detailed information on the cognitive measures in the HRS is available on the HRS Website (http:// hrsonline.isr.umich.edu/docs/userg/Dr-006.pdf).

A trained interviewer assigned proxy respondents to respondents unable to complete the interview, according to study protocol. Each proxy was asked to assess the respondent's memory. Respondents reported to have fair or poor memory were considered to have cognitive impairment. $^{23}$

\section{Disability}

The second eligibility criterion in the Senate's care coordination legislation was having at least one ADL or IADL dependency. The legislation defined ADLs to include eating, toileting, transferring, bathing, dressing, and continence. Eating, toileting, transferring, bathing, and dressing were included in the analysis, but continence was excluded because the toileting variable captures the essence of the disability. The number of respondents reporting ADL dependencies was determined in two steps. First, the number of respondents who reported having difficulty with the ADL because of health or memory problems (difficulty) was determined. Second, the number of respondents who reported having difficulty with and receiving assistance for the ADL (dependency) was determined.
The legislation defined IADLs to include meal preparation, shopping, housekeeping, laundry, money management, telephone use, and transportation use. The HRS does not include questions regarding laundry, and its questions on housekeeping and transportation use do not correspond with the disability targeted by the legislation. In contrast, the HRS includes pertinent questions about the task of taking medications. Thus, the following IADLs were included in the analysis: meal preparation, shopping, money management, telephone use, and taking medications. As with ADLs, the number of respondents reporting IADL dependencies was determined in two steps: first, those who reported having difficulty with the IADL or who could not or did not perform the IADL because of health or memory problems (difficulty), and second, those who reported having difficulty with and receiving assistance for the IADL (dependency).

\section{Demographic Factors}

Demographic variables included age (continuous variable), sex, race (Caucasian, African American, other), marital status (married, unmarried), educational attainment, and net worth. ${ }^{16}$

\section{Care Coordination Eligibility Criteria Sets}

Three care coordination eligibility criteria sets were defined using combinations of complex medical conditions, cognitive impairment, and ADL/IADL difficulty/dependency:

1. 2003 Senate criteria, narrowly interpreted: four complex medical conditions (limited by the activity/severity constraints, including cognitive impairment, excluding hypertension) as the first criterion and at least one ADL or IADL dependency (requiring assistance for the task) as the second criterion.

2. Criteria focused on cognitive impairment: cognitive impairment (regardless of the presence or absence of other conditions) and at least one ADL or IADL dependency (requiring assistance).

3. Criteria based on multiple medical conditions or cognitive impairment: at least three complex medical conditions (limited by the activity/severity constraints, excluding hypertension and cognitive impairment) or cognitive impairment as the first criterion and at least one ADL or IADL dependency (requiring assistance) as the second criterion.

\section{Statistical Analysis}

To adjust for the complex sample design of the HRS, the differential probability of selection, and nonresponse, all analyses were appropriately weighted and adjusted using the statistical package Stata (Release 8.0, Stata Corp., College Station, TX); thus, it was possible to take advantage of the nationally representative data set to produce national population estimates. Standard descriptive methods (frequencies, means, standard deviations) were used in estimating prevalences of respondents with complex medical conditions, cognitive impairment, and disability and the prevalences of respondents meeting criteria set eligibility; in determining confidence intervals; and in making comparisons between subgroups. 


\section{RESULTS}

Figure 1 demonstrates the weighted proportions of respondents who reported a given number of complex medical conditions, examined in three different ways:

- No activity/severity constraints, including hypertension as a condition

- No activity/severity constraints, excluding hypertension as a condition

- Use of activity/severity constraints, excluding hypertension as a condition

Not including hypertension as a complex medical condition decreased the proportion of respondents who reported a given number of conditions. Use of the severity/ activity constraints more markedly decreased these proportions. In the 2000 wave, $15.7 \%$ of respondents had at least four complex medical conditions defined broadly (not limited by activity/severity and including hypertension), whereas only $1.7 \%$ had at least four conditions defined narrowly (limited by activity/severity and excluding hypertension).

Table 1 demonstrates the weighted proportions of respondents who reported specific ADL and IADL difficulties and dependencies and those with a given number of ADL and IADL difficulties and dependencies. Defining ADL and IADL dependency as having difficulty with and requiring personal assistance for the task, compared with only having difficulty with the task, restricted the number of respondents meeting this dependency criterion.

Table 2 shows the weighted proportions of the respondents' demographic characteristics for the 2000 wave (column 1) and for each of the three eligibility criteria sets defined (columns 2-4). Of the total population represented by the 2000 wave, $33 \%$ were aged 65 to 70 , and $21 \%$ were aged 80 and older. Nearly $58 \%$ were female. The women's weighted average age was 75 , and the men's was 74 . Nearly 9\% were African American. Just over $44 \%$ were unmarried.

The first row of Table 2 shows the estimated number of older Americans who would be eligible for the care-coordination benefit based on the eligibility criteria sets defined. Thus, $1.3 \%$ met the narrowly defined Senate eligibility criteria (at least four complex medical conditions (limited by activity/severity, excluding hypertension) and at least one ADL or IADL dependency (requiring assistance)). This corresponds to 427,000 adults aged 65 and older in the United States. (For the broadly defined version, at least four conditions (not limited by activity/severity, including hypertension) and difficulty with at least one ADL or IADL, the percentage increases to $8.3 \%$, representing 2,799,000 older adults.) For the criteria focusing on cognitive impairment, the percentage of respondents with cognitive impairment (regardless of the presence or absence of other conditions) and at least one ADL or IADL dependency was $4.2 \%$. This corresponds to 1,422,000 older Americans. (For the broadly defined version, cognitive impairment and difficulty with at least one ADL or IADL, the percentage increases to $4.6 \%$, representing 1,530,000 older adults.) Last, the criteria based on multiple chronic diseases or cognitive impairment (at least three complex medical conditions (excluding cognitive impairment) or cognitive impairment as the first criterion and at least one ADL or IADL dependency as the second criterion) results in $5.8 \%$, representing 1,969,000 older adults. (For the broadly defined version, at least three conditions (not limited by activity/severity,

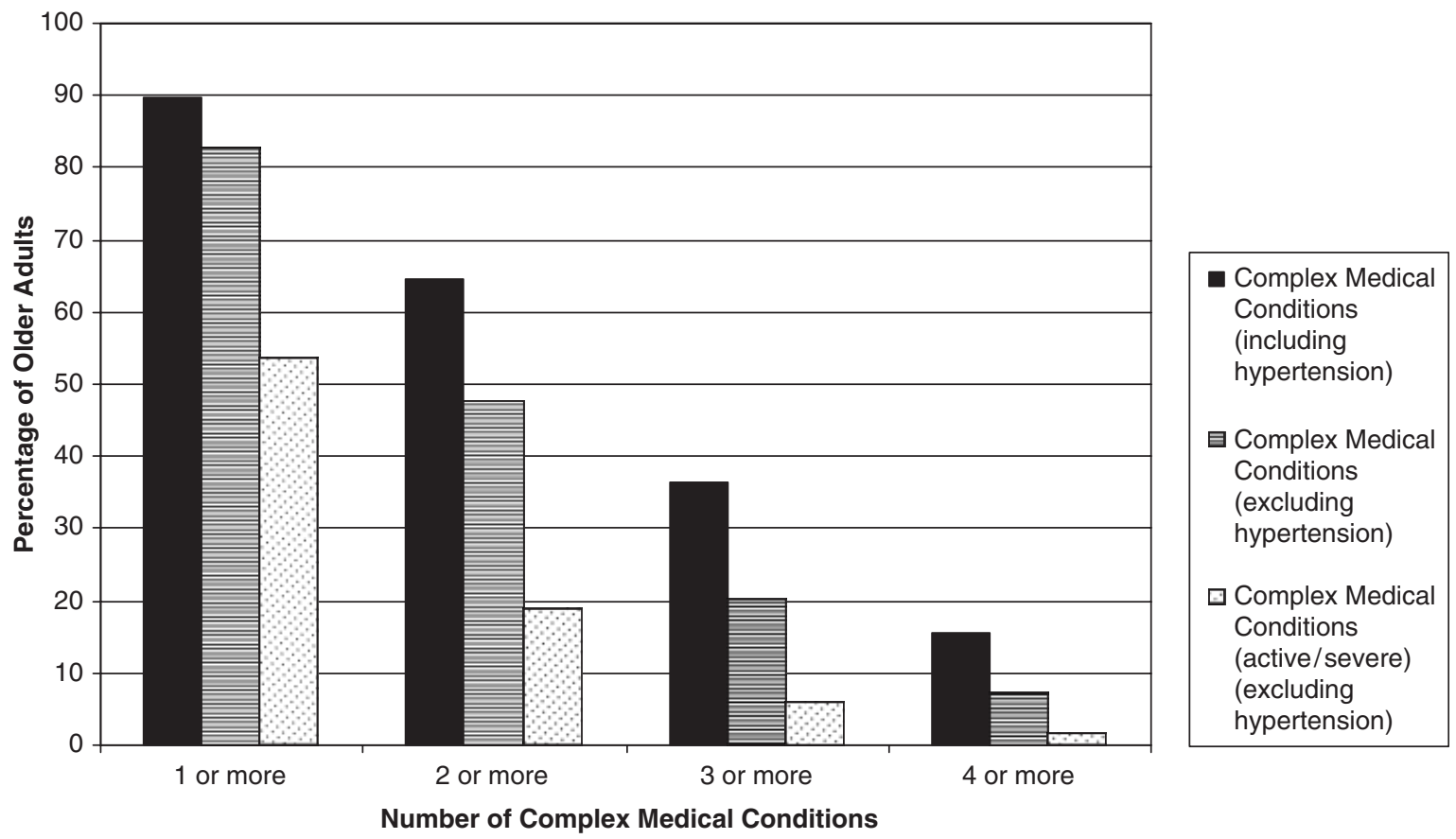

Figure 1. Older adults with number of complex medical conditions. This figure demonstrates the weighted proportions of respondents who reported a given number of complex medical conditions, examined in three different ways: including hypertension as a complex condition, excluding hypertension as a complex condition, and with the use of severity/activity constraints and excluding hypertension as a complex condition. 
Table 1. Weighted Proportion (\%) of Respondents' with Activity of Daily Living (ADL)/Instrumental Activity of Daily Living (IADL) Difficulty/Dependency

\begin{tabular}{|c|c|c|}
\hline ADLs/IADLs & Difficulty & $\begin{array}{l}\text { Dependency } \\
\text { (Assistance) }\end{array}$ \\
\hline \multicolumn{3}{|l|}{ Individual ADLs } \\
\hline Bathing & 9.0 & 5.0 \\
\hline Dressing & 10.9 & 5.1 \\
\hline Eating & 3.5 & 2.3 \\
\hline Transferring & 8.7 & 2.1 \\
\hline Toileting & 6.4 & 1.7 \\
\hline \multicolumn{3}{|l|}{ Individual IADLs } \\
\hline Grocery shopping & 10.9 & 9.5 \\
\hline Meal preparation & 7.4 & 5.7 \\
\hline Taking medication & 3.8 & 2.9 \\
\hline Managing money & 7.0 & 6.3 \\
\hline Phone & 5.1 & 3.5 \\
\hline \multicolumn{3}{|l|}{ Number of ADLs } \\
\hline$\geq 1$ & 19.7 & 7.9 \\
\hline$\geq 2$ & 9.6 & 3.9 \\
\hline$\geq 3$ & 5.4 & 2.2 \\
\hline$\geq 4$ & 2.9 & 1.5 \\
\hline \multicolumn{3}{|l|}{ Number of IADLs } \\
\hline$\geq 1$ & 16.3 & 13.3 \\
\hline$\geq 2$ & 8.6 & 7.1 \\
\hline$\geq 3$ & 4.8 & 4.1 \\
\hline$\geq 4$ & 2.9 & 2.3 \\
\hline \multicolumn{3}{|c|}{ Number of ADLs and/or IADLs } \\
\hline$\geq 1$ & 25.6 & 15.3 \\
\hline$\geq 2$ & 15.0 & 8.9 \\
\hline$\geq 3$ & 10.4 & 6.3 \\
\hline$\geq 4$ & 7.3 & 4.6 \\
\hline
\end{tabular}

including hypertension, excluding cognitive impairment) or cognitive impairment as the first criterion and at least one ADL or IADL difficulty as the second criterion, the percentage increases to $16.6 \%$, representing $5,580,000$ older adults.)

Table 2 also compares the demographic characteristics of the older adults meeting the different criteria sets with those of the total population. Those meeting the narrowly defined Senate eligibility criteria (column 2) were more likely to be older, African American, and unmarried. The age distribution of those meeting the cognitive criteria set is shifted toward a more-advanced age, reflecting the age distribution of cognitive impairment. In general, all criteria sets identify individuals who are older, from an ethnic minority group, and unmarried; these individuals are also less educated and have a lower net worth (data not shown).

Figure 2 illustrates the overlap of complex medical conditions, cognitive impairment, and ADL/IADL dependency in the older adult population. Figure $2 \mathrm{~A}$ demonstrates the overlap of those having at least four complex medical conditions (one of which may be cognitive impairment) with those having at least one ADL or IADL dependency; these are the criteria in the original Senate legislation. Thus, $1.7 \%$ of respondents have at least four complex medical conditions, $15.3 \%$ have at least one ADL or IADL dependency, and $1.3 \%$ satisfy both criteria. In contrast, Figure $2 \mathrm{~B}$ demonstrates the overlap of those having at least three complex medical conditions (excluding cognitive impairment) with those having cognitive impairment and with those having at least one ADL or IADL dependency; these are the criteria in the final eligibility criteria set. Here, $4.6 \%$ have at least three complex medical conditions, $6.1 \%$ have cognitive impairment, and $15.3 \%$ have at least one ADL or IADL dependency; $5.8 \%$ satisfy the combination of criteria.

\section{DISCUSSION}

This study estimated the number and characteristics of older adults who would meet different sets of clinically meaningful eligibility criteria for a Medicare care-coordination benefit. These criteria sets were derived in part from previous legislative attempts to define eligibility criteria, most recently the Senate version of the Medicare Modernization Act; they were also derived by considering the defining clinical characteristics of older adults: chronic diseases, cognitive impairment, and personal disability. Using combinations of these characteristics, it was demonstrated that different criteria sets yielded greater or lesser numbers of eligible older adults. This research also identifies different groups of older adults who could be matched to different healthcare delivery models.

The three criteria sets that were chosen are related: criteria sets 1 and 3 emphasize medical conditions, criteria sets 2 and 3 emphasize cognitive impairment, and criteria set 3 roughly combines sets 1 and 2 . The more-restrictive criteria set 1 has a greater proportion of individuals who are African American, less educated, and relatively poor. Criteria sets 2 and 3, emphasizing cognitive impairment, select an older group.

A criterion requiring multiple complex medical conditions is included in criteria sets 1 and 3 . There are no widely accepted definitions for the terms "complex medical condition," "chronic condition," and "chronic disease." At times, the terms are used interchangeably; at other times, they are used to indicate distinctions among overlapping categories. The term "complex medical conditions" (following from the language of the Senate legislation) was used in this study to signify chronic diseases (self-reported in the HRS) that may be associated with personal disability.

Furthermore, there is no widely accepted standard for the definition and measurement of chronic diseases in the older adult population. Norms have been developed for measuring chronic diseases and assessing their severity from administrative claims data from Medicare and other insurers. ${ }^{24}$ Yet claims data have disadvantages. These data may only represent individuals who use health services. Major diseases associated with cognitive impairment, such as stroke or Alzheimer's, can be found in claims data, but these are often not the diseases that generate a medical encounter, and thus their prevalence may be underestimated. Also, claims data do not include associated disability measures.

Self-report data are a second source of information about chronic diseases, and self-report data from large health interview surveys are often used in health policy research. ${ }^{1,21,25}$ Although this type of data lacks many of the disadvantages inherent to claims data, potential problems exist. It can be unclear which diseases to include as "chronic diseases." For example, risk factors for atherosclerotic 
Table 2. Respondents' Demographic Characteristics

\begin{tabular}{|c|c|c|c|c|}
\hline \multirow[b]{2}{*}{ Characteristic } & \multirow[b]{2}{*}{$\begin{array}{c}2000 \text { Wave } \\
(\mathrm{N}=33.6 \text { million }) \%\end{array}$} & $\begin{array}{l}\text { Eligibility Criteria } 1 * \\
(\mathrm{n}=427,000 ; 1.3 \%)\end{array}$ & $\begin{array}{c}\text { Eligibility Criteria } 2^{\dagger} \\
(\mathrm{n}=1,422,000 ; 4.2 \%)\end{array}$ & $\begin{array}{c}\text { Eligibility Criteria } 3^{\ddagger} \\
(n=1,969,000 ; 5.8 \%)\end{array}$ \\
\hline & & \multicolumn{3}{|c|}{$\begin{array}{c}\% §(95 \% \text { Confidence } \\
\text { Interval) }\end{array}$} \\
\hline \multicolumn{5}{|l|}{ Age } \\
\hline $65-70$ & 33.1 & $20.9(14.9-28.4)$ & $13.4(10.0-17.7)$ & 16.8 (13.8-20.3) \\
\hline$>80$ & 21.3 & $31.5(24.3-39.7)$ & $48.6(44.2-53.1)$ & $42.8(39.2-46.4)$ \\
\hline \multicolumn{5}{|l|}{ Sex } \\
\hline Male & 42.4 & $43.7(35.2-52.7)$ & $43.2(38.8-47.7)$ & $39.1(35.6-42.8)$ \\
\hline Female & 57.6 & $56.3(47.3-64.8)$ & $56.8(52.3-61.2)$ & $60.9(57.2-64.4)$ \\
\hline \multicolumn{5}{|l|}{ Race } \\
\hline Unmarried & 44.3 & $50.4(40.9-59.9)$ & $54.0(48.7-59.2)$ & $55.6(51.1-60.0)$ \\
\hline
\end{tabular}

disease, such as hypertension and hyperlipidemia, although treated medically, are not themselves usually associated with morbidity. Many chronic diseases (e.g., peripheral neuropathy, Parkinson's) are underdiagnosed, and unaware of them, respondents do not report them. In this respect, cognitive impairment and the diseases associated with it pose a special challenge when using self-report data to study diseases in older adults. Also, some chronic diseases (e.g., chronic rhinitis, irritable bowel disease) are arguably less important.

This research included common chronic diseases, grouped cardiac diseases and procedures, grouped musculoskeletal conditions and procedures, and included and excluded hypertension. The performance-based determination of cognitive impairment in the HRS ${ }^{22}$ was made use of, and so respondents' self-report of a dementia diagnosis was not relied on. ${ }^{26}$ This research further ascribed an activity/ severity constraint to each disease condition, primarily by limiting conditions to those requiring ongoing treatment. Although this can only be an approximate estimate of severity, it permits comparison with nationally reported disease prevalences with and without severity constraints. These findings are generally consistent with other published research using the HRS and other data sources. ${ }^{1,21,27}$ For example, using Medicare claims data and not adjusting for disease activity or severity, it was found that $60 \%$ of Medicare beneficiaries have two or more diseases; ${ }^{1}$ these findings are consistent with the results of the current study that $65.2 \%$ of adults aged 65 and older have at least two chronic diseases (not adjusting for severity).

Cognitive impairment is another defining clinical characteristic of older adults; it is not only related to neurological diseases but also affects the ability of older individuals to manage their other chronic diseases. ${ }^{21}$ Policy-makers are beginning to address cognitive impairment when considering care coordination schemes; recent legislative language allows for dementia to be one of the chronic diseases. Because cognitive impairment is underevaluated, and because it affects self-management of all other chronic diseases, criteria sets ( 2 and 3 ) were tested in which cognitive impairment, directly measured in the data, was explicitly addressed.

A criterion requiring personal disability is included in all three of the eligibility criteria sets. Measurement of ADL/ IADL dependency is less problematic than that of chronic diseases and cognitive impairment. Depending on the research question, ADL and IADL task limitations can be measured in terms of difficulty or of need for assistance, and prevalences for each method have been reported. ADL difficulty and dependency trends in older adults were recently examined, comparing five national data sets, including the HRS. ${ }^{28}$ This research differed in that it included incontinence as an ADL, included long-term care facility residents, and excluded adults aged 65 to 71 . Taking these differences into account, the ADL difficulty and dependency results are comparable with those of the current study.

Current Medicare reforms include attempts to define and test models to provide and pay for care for patients with multiple complex medical conditions and associated disability. In the commercial insurance market, the disease management model is considered to be promising for those with a single disease that dominates their healthcare utilization, ${ }^{29}$ although problems have been noted. Many studies are short term and performed in middle-aged populations in managed care settings. ${ }^{2,3}$ The disease management model may be inadequate or break down in the older-adult, 


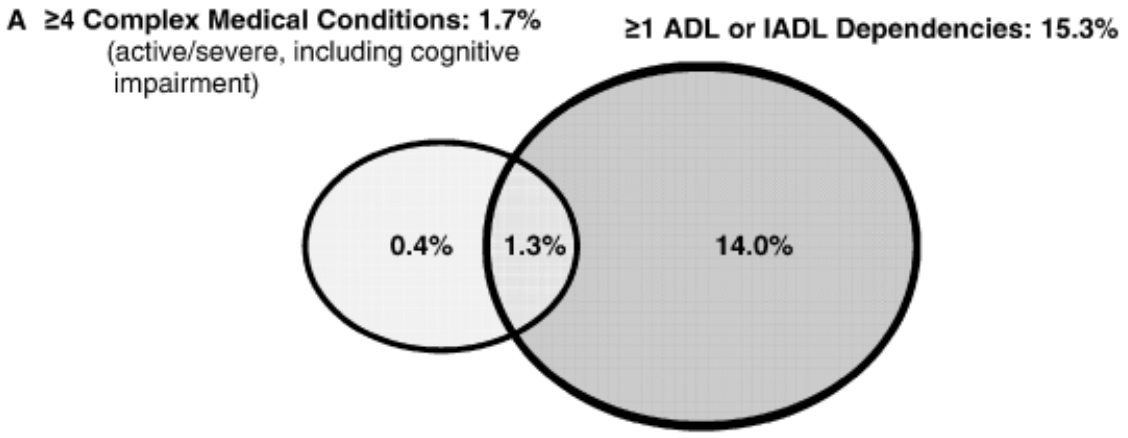

B
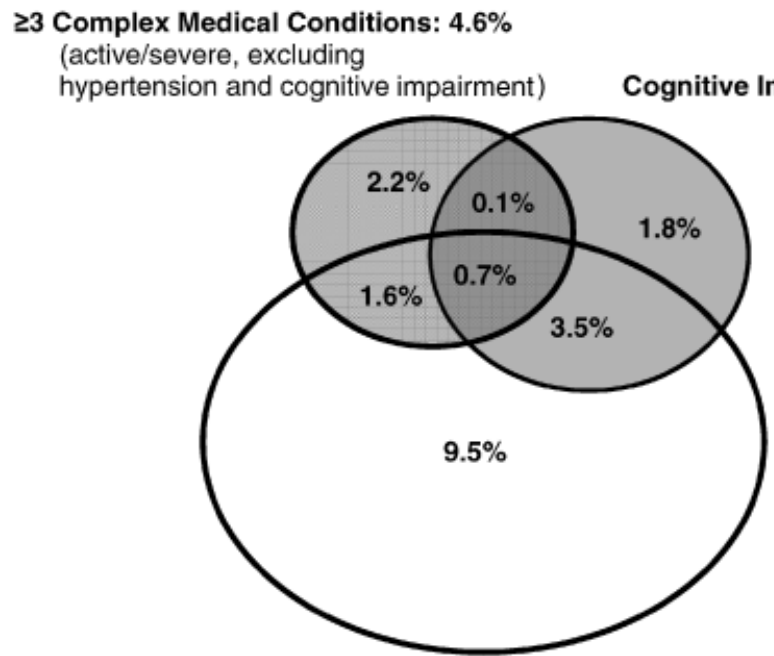

$\geq 1$ ADL or IADL Dependencies: $\mathbf{1 5 . 3} \%$

Figure 2. Overlap of complex medical conditions, cognitive impairment, and activity of daily living/instrumental activity of daily living (ADL/IADL) dependency. A. Proportions of adults aged 65 and older with complex medical conditions and ADL/IADL dependencies. Overlap of those having at least four complex medical conditions with those having at least one ADL or IADL dependency; these are the criteria in the original Senate legislation. B. Proportions of adults aged 65 and older with complex medical conditions, cognitive impairment, and ADL/IADL dependencies. Overlap of those having at least three complex medical conditions (excluding cognitive impairment) with those having cognitive impairment and with those having at least one ADL or IADL dependency.

fee-for-service Medicare population, where multiple chronic diseases are common and where utilization is related not to a single dominant disease but to multiple diseases that affect one another. ${ }^{30}$

It is not clear how to design the best care delivery and payment mechanism for the subset of Medicare patients with a combination of chronic diseases, cognitive impairment, and disability, many of whom are high users of health care. The care-coordination model has been proposed to improve quality by coordinating the care of vulnerable patients across the spectrum of multiple care providers and settings and to decrease utilization by improving appropriateness and decreasing duplication of care. ${ }^{6}$

For both disease management and care-coordination models, there is little information about how to target older adults who would most benefit from them. Previous studies examining social models of care coordination in the 1990s attributed their failure to affect care efficiency to poor patient targeting. ${ }^{6-9,31}$ In contrast to a focus on coordination of medical care by physicians, the goal of the social model of care coordination was to provide home care services by a spectrum of providers. Also, the populations that the two models of care coordination aimed to serve overlap but are distinct. The original Senate version of the Medicare Modernization Act required eligible beneficiaries to have at least four complex medical conditions (one of which could be cognitive impairment) and at least one ADL or IADL impairment. The social model of care coordination was directed at those with long-term disability, cognitive impairment, or both; addressing multiple medical conditions was not a purpose, and having multiple conditions was not an eligibility criterion.

Despite these differences between medical care coordination and social care coordination, it is instructive to examine the research on eligibility criteria for the latter. Two studies used the 1984 National Long-Term Care Survey to estimate proportions of older adults having combinations of ADL disability and cognitive impairment and thus eligible for some form of home care. ${ }^{32,33}$ One determined that $6.1 \%$ of adults aged 65 and older had one or more ADL dependencies; $3.3 \%$, two or more dependencies; and $2.1 \%$, three or more dependencies (compare with Table 1). ${ }^{32}$ Depending on the number of ADL dependencies required and the strictness of the cognitive impairment criterion, it esti- 
mated that $3.9 \%$ to $9.0 \%$ would have the long-term disability or the cognitive impairment to be eligible for the home care benefit. In comparison with the current legislation, eligibility criteria set 2 is most analogous, requiring at least one ADL or IADL dependency and cognitive impairment, yielding $4.2 \%$ of adults aged 65 and older (Figure 2B).

Both studies make observations regarding the social model of care coordination that are applicable to the medical model. $32-34$ First, the strictness or expansiveness of the eligibility criteria has a sizable effect on the number of older adults eligible for the benefit and thus its costs. Second, much depends on how administrative and regulatory bodies interpret the legislative language in its actual implementation.

The current study suggests some strategies to improve patient targeting. The healthcare utilization and costs associated with individuals meeting different eligibility criteria sets can be studied. Characteristics of people within these groups could be matched to a service delivery model. For instance, as Figure 2B demonstrates, a sizable proportion $(9.5 \%)$ of older adults have dependency without dementia and without three or more diseases; this may be a group of older adults with one or two diseases that affect their function. Such patients may benefit from disease management, which would address the effect of that disease on their quality of life and disease self-management abilities. In contrast, the group with multiple diseases, cognitive impairment, and disability is likely to need multidisciplinary care coordination to address medical and personal care needs. This is the group to target for a Medicare care-coordination benefit. Finally, the group with multiple chronic diseases and without disability may benefit most from assistance in navigating the medical system and managing multiple medications. To translate these potential groupings into clinically relevant targeting and care delivery programs, future research on targeting methodologies and outcomes is needed.

Care coordination as a healthcare delivery model is being advanced in an environment of increasing numbers of Medicare beneficiaries, the aging of the beneficiary population, and continuing healthcare inflation. In this environment, the healthcare utilization and costs associated with individuals meeting different eligibility criteria sets are relevant to the targeting of the benefit and its effect on the overall costs of these beneficiaries. Future research examining the cross-sectional and longitudinal costs of beneficiaries is needed. This will provide an indication of whether savings elsewhere can offset the cost of the benefit such that the overall cost of care is unchanged or decreased.

Although not a focus of this study, the topic of healthcare delivery models for older adults with multiple medical conditions includes the matter of who will provide the care. The legislation specifies physicians as providers. Presumably, most will be primary care physicians, internists, and family practitioners, with involvement also of geriatric nurse practitioners and some specialists. The number and distribution of geriatricians is such that they alone would be unable to meet the need.

The key strength of this research is that it is based on a large, nationally representative survey (HRS) that provides data on chronic diseases, cognitive impairment, and ADL and IADL dependencies. The HRS survey includes questions that enable the activity or severity of the medical condition or the dependency to be characterized, and it includes a performance-based measure of cognitive impairment. Finally, HRS is a biennial longitudinal survey that includes utilization and cost data, making possible future studies that examine the cross-sectional and longitudinal association between complex medical conditions, cognitive impairment, and disability and health, utilization, and cost outcomes.

This study has several potential limitations. First, HRS is based on self-report data. In particular, the activity/severity constraint developed for complex medical conditions is based on self-report data and may occasionally be problematic. For example, use of the constraint "remaining problems" for the stroke condition potentially overlaps with the ADL/IADL dependency criterion. Second, the questions included in the HRS survey limited the complex medical conditions chosen for this study. The 2000 wave does not include specific questions on diseases such as chronic kidney disease and Parkinson's. Third, the questions included in the HRS survey likewise limited the ADL and IADL dependencies chosen for this study. Fourth, a Medicare care-coordination benefit would apply to all Medicare beneficiaries, regardless of age, but this study excludes adults younger than 65 . Some younger beneficiaries (for example, those with end-stage renal disease and disability), would likely meet potential criteria. Finally, this study also excluded people residing in nursing facilities at the time of their interview. These most likely were "long term" nursing facility residents and therefore not eligible for the care-coordination benefit, yet it is possible that some excluded respondents were "subacute stay" nursing facility residents who might be eligible for the benefit upon their discharge from the nursing facility.

The problems of multiple chronic diseases, cognitive impairment, and personal disability are of increasing importance for older Medicare beneficiaries and must be addressed in any Medicare reform. Although only a small proportion of older beneficiaries have these problems, these beneficiaries experience increased healthcare utilization, fragmented care, and potentially poor quality of care. Designing a care-coordination benefit begins with the identification of beneficiaries who are likely to experience improved care as a result of care coordination. This research has proposed several possible eligibility criteria sets and described those older adults who would be eligible under different scenarios, setting the stage for future research to identify the health outcomes and costs of these proposed groups.

\section{ACKNOWLEDGMENTS}

The authors gratefully acknowledge Tisha L. Moore for her assistance with manuscript preparation and development of figures and table.

Financial Disclosure: None of the authors have received financial support from consultantships or speakers forum; nor do they have any company holdings (e.g., stocks) or patents. None have conflicts of interest or financial arrangements with a company whose product is associated with the submitted manuscript.

Author Contributions: Christine T. Cigolle: data analysis and interpretation, manuscript preparation and editing. 
Kenneth M. Langa: data interpretation, manuscript editing. Mohammed U. Kabeto: data analysis. Caroline S. Blaum: data interpretation, manuscript preparation and editing.

Sponsor's Role: There was no sponsor for this article.

\section{REFERENCES}

1. Wolff JL, Starfield B, Anderson G. Prevalence, expenditures, and complications of multiple chronic conditions in the elderly. Arch Intern Med 2002;162:2269-2276.

2. Weingarten SR, Henning JM, Badamgarav E et al. Interventions used in disease management programmes for patients with chronic illness-which ones work? Meta-analysis of published reports. BMJ 2002;325:925.

3. Bodenheimer T. Disease management in the American market. BMJ 2000;320:563-566.

4. Braunstein JB, Anderson GF, Gerstenblith G et al. Noncardiac comorbidity increases preventable hospitalizations and mortality among Medicare beneficiaries with chronic heart failure. J Am Coll Cardiol 2003;42:1226-1233.

5. Niefeld MR, Braunstein JB, Wu AW et al. Preventable hospitalization among elderly Medicare beneficiaries with type 2 diabetes. Diabetes Care 2003;26:1344-1349.

6. Capitman J. Effective coordination of medical and supportive services. J Aging Health 2003;15:124-164.

7. Chernew ME, Weissert WG, Hirth RA. Heterogeneity of risk in a managed home health care population. Med Care 2001;39:1002-1013.

8. Weissert W, Chernew M, Hirth R. Beyond managed long-term care. Paying for home care based on risk of adverse outcomes. Health Aff (Millwood) 2001;20:172-180

9. Weissert W, Chernew M, Hirth R. Titrating versus targeting home care services to frail elderly clients. An application of agency theory and cost-benefit analysis to home care policy. J Aging Health 2003;15:99-123.

10. United States Senate, 108th Congress. Geriatric Care Act of 2003. February 13, 2003 [on-line]. Available at http://thomas.loc.gov/cgi-bin/query/ D?c108:1./temp/ c108CCrHbY Accessed June 29, 2005.

11. United States House of Representatives, 108 th Congress. Geriatric Care Act of 2003. January 7, 2003 [on-line]. Available at http://thomas.loc.gov/cgi-bin/ query/D?c108:2./temp/ c108CCrHbY Accessed June 29, 2005.

12. United States Senate, 108th Congress. Medicare Chronic Care Improvement Act of 2003, June 4, 2003 [on-line]. Available at http://thomas.loc.gov/cgi-bin/ query/D?c108:3./temp/ c108CCrHbY Accessed June 29, 2005.

13. United States House of Representatives, 108th Congress. Medicare Chronic Care Improvement Act of 2003. June 4, 2003 [on-line]. Available at http:// thomas.loc. gov/cgi-bin/query/D?c108:4./temp/ c108CCrHbY Accessed June $29,2005$.

14. United States Senate, 108th Congress. Prescription Drug and Medicare Improvement Act of 2003. June 11, 2003 [on-line]. Available at http:// thomas.loc.gov/cgi-bin/query/F?c108:22./temp/ c108CCrHbY:e716993 Accessed June 29, 2005.
15. Soldo BJ, Hurd MD, Rodgers WL et al. Asset and Health Dynamics Among the Oldest Old: An overview of the AHEAD Study. J Gerontol B Psychol Sci Soc Sci 1997;52 Spec No:1-20.

16. Juster FT, Suzman R. An overview of the Health and Retirement Study. J Hum Resour 1995;30:S7-S56.

17. Boult C, Kane RL, Louis TA et al. Chronic conditions that lead to functional limitation in the elderly. J Gerontol 1994;49:M28-M36.

18. Tinetti ME, Fried T. The end of the disease era. Am J Med 2004;116:179-185.

19. Tinetti ME, Inouye SK, Gill TM et al. Shared risk factors for falls, incontinence, and functional dependence. Unifying the approach to geriatric syndromes. JAMA 1995;273:1348-1353.

20. Folstein MF, Folstein SE, McHugh PR. 'Mini-mental state'. A practical method for grading the cognitive state of patients for the clinician. J Psychiatr Res 1975;12:189-198.

21. Blaum CS, Ofstedal MB, Liang J. Low cognitive performance, comorbid disease, and task-specific disability: Findings from a nationally representative survey. J Gerontol A Biol Sci Med Sci 2002;57A:M523-M531.

22. Herzog AR, Wallace RB. Measures of cognitive functioning in the AHEAD Study. J Gerontol B Psychol Sci Soc Sci 1997;52 Spec No:37-48.

23. Langa KM, Chernew ME, Kabeto MU et al. National estimates of the quantity and cost of informal caregiving for the elderly with dementia. J Gen Intern Med 2001;16:770-778.

24. Starfield B, Weiner J, Mumford L et al. Ambulatory care groups. A categorization of diagnoses for research and management. Health Serv Res 1991;26:53-74.

25. Simpson CF, Boyd CM, Carlson MC et al. Agreement between self-report of disease diagnoses and medical record validation in disabled older women: Factors that modify agreement. J Am Geriatr Soc 2004;52: 123-127.

26. Cigolle C, Kabeto M, Langa $\mathrm{K}$ et al. Setting eligibility criteria for a care coordination benefit (Abstract). Gerontologist 2003;43:428.

27. Fried LP, Bandeen-Roche K, Kasper JD et al. Association of comorbidity with disability in older women: The Women's Health and Aging Study. J Clin Epidemiol 1999;52:27-37

28. Freedman VA, Crimmins E, Schoeni RF et al. Resolving inconsistencies in trends in old-age disability: Report from a technical working group. Demography 2004;41:417-441.

29. Casalino LP. Disease management and the organization of physician practice. JAMA 2005;293:485-488.

30. Bodenheimer T. Disease management - promises and pitfalls. N Engl J Med 1999;340:1202-1205.

31. Weissert WG, Hirth RA, Chernew ME et al. Case management. Effects of improved risk and value information. Gerontologist 2003;43:797-805.

32. Jackson ME, Burwell B, Clark RF et al. Eligibility for publicly financed home care. Am J Public Health 1992;82:853-856.

33. Spector WD. Cognitive impairment and disruptive behaviors among community-based elderly persons: Implications for targeting long-term care. Gerontologist 1991;31:51-59.

34. Spector WD, Kemper P. Disability and cognitive impairment criteria: Targeting those who need the most home care. Gerontologist 1994;34:640-651. 